\title{
Dialkali-Metal Monochalcogenide Semiconductors with High Mobility and Tunable Magnetism
}

\author{
Chenqiang Hua, ${ }^{1,2}$ Feng Sheng, ${ }^{1}$ Qifeng $\mathrm{Hu},{ }^{1}$ \\ Zhu-An Xu, ${ }^{1,3}$ Yunhao Lu, ${ }^{2, *}$ and Yi Zheng, ${ }^{1,3, \dagger}$ \\ ${ }^{1}$ Department of Physics, Zhejiang University, Hangzhou 310027, P. R. China \\ ${ }^{2}$ State Key Lab of Silicon Materials, \\ School of Materials Science and Engineering, \\ Zhejiang University, Hangzhou 31002\%, P. R. China \\ ${ }^{3}$ Collaborative Innovation Centre of Advanced Microstructures, \\ Nanjing University, Nanjing 210093, P. R. China
}

(Dated: August 28, 2018)

\begin{abstract}
The discovery of archetypal two-dimensional (2D) materials provides enormous opportunities in both fundamental breakthroughs and device applications, as evident by the research booming in graphene, atomically thin transition-metal chalcogenides, and few-layer black phosphorous in the past decade. Here, we report a new, large family of semiconducting dialkali-metal monochalcogenides (DMMCs) with an inherent $\mathrm{A}_{2} \mathrm{X}$ monolayer structure, in which two alkali sub-monolayers form hexagonal close packing and sandwich the triangular chalcogen atomic plane. Such unique lattice structure leads to extraordinary physical properties, such as good dynamical and thermal stability, visible to near-infrared light energy gap, high electron mobility (e.g. $1.87 \times 10^{4} \mathrm{~cm}^{2} \mathrm{~V}^{-1} \mathrm{~S}^{-1}$ in $\mathrm{K}_{2} \mathrm{O}$ ). Most strikingly, DMMC monolayers (MLs) host extended van Hove singularities near the valence band $(\mathrm{VB})$ edge, which can be readily accessed by moderate hole doping of $\sim 1.0 \times 10^{13}$ $\mathrm{cm}^{-2}$. Once the critical points are reached, DMMC MLs undergo spontaneous ferromagnetic transition when the top VBs become fully spin-polarized by strong exchange interactions. Such gate tunable magnetism in DMMC MLs are promising for exploring novel device concepts in spintronics, electronics and optoelectronics.
\end{abstract}

\footnotetext{
*luyh@zju.edu.cn

$\dagger$ phyzhengyi@zju.edu.cn
} 


\section{INTRODUCTION}

Although the critical importance of dimensionality in determining the extraordinary physical properties of low-dimension systems has long been recognized since Richard Feyman, the groundbreaking experiments on graphene [1-3] provide a fascinating platform for exploring exotic phenomena in a rather simple hexagonal lattice. After the gold rush of graphene, the search of new two-dimensional (2D) systems have received unparalleled attention in the hope of novel physical properties and prototype functionalities. However, despite the flourishing of 2D materials [4-10], the majority of 2D researches remain heavily focusing on the archetypal systems of graphene [11], transition-metal dichalcogenides (TMDCs) [12 15], and few-layer black phosphorus (BP) [16, 17]. These 2D paradigms share the common features of weak interlayer coupling by van der Waals interactions, good thermal stability at room temperature, and high charge carrier mobility. Nevertheless, very recently, there are emergent 2D materials with tempting physical properties beyond the aforementioned systems, such as the InSe family [18, 19], 2D ferromagnetic van der Waals crystals [20, 21], binary maingroup compounds with the BP-type puckering lattice [22 24], and the huge family of carbide and nitride based transition metal MXenes, in which M represents transition metal cation and $\mathrm{X}$ is $\mathrm{C}$ or $\mathrm{N}$ anion, respectively [8, 25].

Here, by using ab initio density functional calculations, we discover a large family of dialkali-metal monochalcogenides (DMMCs) with excellent dynamical and thermal stability, visible to near-infrared light energy gap, and very high electron mobility of exceeding $1.0 \times$ $10^{3} \mathrm{~cm}^{2} \mathrm{~V}^{-1} \mathrm{~s}^{-1}$. With the same lattice structure of $1 \mathrm{~T}-\mathrm{TMDCs}$, DMMCs have the inherent layer-by-layer structure with very weak interlayer coupling, due to the intrinsic +1 oxidation state of alkali cations. All these features make DMMCs a unique choice beyond TMDCs for fundamental studies of 2D systems and for developing potential electronic and optoelectronic devices. Most fascinatingly, all DMMC monolayers (MLs) host extended singularity points in the density of states near the Mexican-hat shaped valence band maximum (VBM). Using hole doping in the range of $\sim 1.0 \times 10^{13}-2.5 \times 10^{13} \mathrm{~cm}^{-2}$ via electrostatic or liquid ion gating, spontaneous ferromagnetic transitions, which lead to half-metallic character with full spin polarized top valence bands, can be triggered by strong exchange interactions in these 2D systems. Such gate tunable magnetism and half metallicity in DMMC MLs may pave new routes in novel device concepts for spintronics. 


\section{RESULTS AND DISCUSSION}

\section{A. Structure and Stability}

Dialkali-metal monochalcogenides form a large family with sixteen compounds in the general formula of $\mathrm{A}_{2} \mathrm{X}$, where $\mathrm{A}$ represents an alkali atom ( $\mathrm{Na}, \mathrm{K}, \mathrm{Rb}$ or $\mathrm{Cs}$ ) and $\mathrm{X}$ is a chalcogen anion of $\mathrm{O}, \mathrm{S}, \mathrm{Se}$ or Te. The synthesis of bulk dicesium monoxide $\left(\mathrm{Cs}_{2} \mathrm{O}\right)$ was first reported in 1955 [26] with the space group $R \overline{3} m$ (No. 166), which has the same lattice structures as the well-known 1T-TMDCs [27] as displayed in Fig. 17a and 2a. By taking van der Waals (vdW) corrections into account, the optimized lattice constants of bulk $\mathrm{Cs}_{2} \mathrm{O}$ are $a=4.23 \AA, b=4.23 \AA$, and $c=19.88 \AA$, respectively, which are in excellent agreement with the experimental values [26]. In the $2 \mathrm{D}$ limit, the monolayer of DMMCs has the space group $P \overline{3} m 1$ (No. 164). The primitive unit cell consists of two alkali cations and one group-VIa anions, making $\mathrm{A}_{2} \mathrm{X}$ stoichiometry. The metal-chalcogen ratio in DMMC MLs is distinct from the well-known TMDC MLs, in which the unit cell includes two chalcogenide anions and one metal cation. For instance, in $\mathrm{Cs}_{2} \mathrm{O} \mathrm{ML}$, the centering $\mathrm{O}$ atom is surrounded by six Cs cations, forming a distinctive O-Cs octahedron, as shown in Fig. 11a. Such lattice structure is rooted in strong intralayer O-Cs ionic bonding, as revealed by the analysis of electron localization functions (ELFs). As shown in Fig. 1 b, the electron densities are highly localized around $\mathrm{Cs}$ and $\mathrm{O}$ atoms with negligible inter-atom distribution, reflecting ionic bonding and electron donation from Cs to $\mathrm{O}$ atoms. The calculated oxidation state of $\mathrm{O}$ anions, as represented by the Hirshfeld charge, is nearly identical to that of $\mathrm{BaO}$, also confirming the dianionic character of oxygen in $\mathrm{Cs}_{2} \mathrm{O}$. No accumulation of electron density is observed between Cs atoms, suggesting no chemical bond between them. The A-X octahedral geometry centring the chalcogen dianion is general observed for the whole family, as presented in Fig. 1 $1 \mathrm{~b}$ for $\mathrm{Na}_{2} \mathrm{O}$ ML and in Supplementary Information (SI) Figure S1-S4 for the other 14 DMMC MLs.

Due to the intrinsic full oxidation state $(+1)$ of Cs, the interlayer coupling between Cs subMLs is very weak, which only introduces marginal changes to the in-plane lattice constants. The optimized $a$ and $b$ of $\mathrm{Cs}_{2} \mathrm{O}$ ML $(4.26 \AA)$ are almost the same as the experimental bulk

value. Comparing with the paradigmatic 2D systems of $\mathrm{MoS}_{2}$ and graphite, we find that there are even less interlayer electron density localization in $\mathrm{Cs}_{2} \mathrm{O}$ as a result of dominant 
in-plane ionic bonding, which also means micromechanical exfoliation of $\mathrm{Cs}_{2} \mathrm{O}$ crystals can be readily achieved (Fig. 2a). Indeed, we calculate the exfoliation energy of $\mathrm{Cs}_{2} \mathrm{O}$ ML to be $\sim 0.19 \mathrm{~J} / \mathrm{m}^{2}$ (Fig. 2 $\mathrm{b}$ ), which is surprisingly lower than the value of $0.33 \mathrm{~J} / \mathrm{m}^{2}$ for graphene [28]. It has to been emphasized here that the lower exfoliation energy of bulk $\mathrm{Cs}_{2} \mathrm{O}$, compared with $\mathrm{MoS}_{2}\left(\sim 0.42 \mathrm{~J} / \mathrm{m}^{2}\right)$ and graphite, is consistently obtained by different vdW correction methods, which is summarized in SI Table S1.

To assess the dynamical stability, which is also crucial for the micro-exfoliation of $\mathrm{Cs}_{2} \mathrm{O}$ ML, we have computed the phonon dispersion with the finite displacement method. As shown in Fig. 22, the good stability of $\mathrm{ML} \mathrm{Cs}_{2} \mathrm{O}$ is evident by the positive values of all phonon modes. We further check the room-temperature (RT) thermal stability of $\mathrm{Cs}_{2} \mathrm{O}$ ML by firstprinciple molecular dynamics simulations. The fluctuation of energy and temperature as a function of time are plotted in Fig. $2 \mathrm{~d}$. After running 4000 steps (10 ps), the $3 \times 3 \times 1$ trigonal lattice is well sustained, and the free energy of the supercell converges. Excellent dynamical and thermal stability have also been validated for the other DMMC MLs (See SI Figure S5 and S6). Thus, it is feasible to experimentally exfoliate DMMC MLs for device fabrications and applications.

\section{B. Electronic Properties}

We now elucidate the electronic structures and fundamental physical properties of DMMC MLs using two representative examples of $\mathrm{Cs}_{2} \mathrm{O}$ and $\mathrm{Na}_{2} \mathrm{O}$ MLs. As shown in Fig. $3 \mathrm{a}, \mathrm{Na}_{2} \mathrm{O}$ ML is a 2D semiconductor with a direct bandgap of $\sim 1.99 \mathrm{eV}$ at the $\Gamma$ point. Noticeably, the VBM of $\mathrm{Na}_{2} \mathrm{O}$ ML is mainly contributed by $\mathrm{O}-2 p$ orbitals (its projection weight is not shown in the energy dispersion for clarity) hybridized with very low weight of Na- $2 p$

(weighted by green triangles), while the CBM is dominated by Na-3s (red squares). The electronic band structure undergoes a pronounced change when $\mathrm{Na}$ is replaced by $\mathrm{Cs}$, whose strongly delocalized $5 p$ orbitals induce significant density of states (DOS) away from the $\Gamma$ point, which is still dominated by O- $2 p$. As the energy of Cs- $5 p$ orbitals is $\sim 0.4 \mathrm{eV}$ higher than the $2 p$ orbitals of $\mathrm{O}$, the inter $p$-orbital hybridization reverses the energy levels of VB1 and VB2 along the $\Gamma-M$ direction. The resulting $\mathrm{Cs}_{2} \mathrm{O}$ ML is an indirect semiconductor with multiple VBMs centering the $M$ points (Fig. 3b). In general, for heavier cations of $\mathrm{K}, \mathrm{Rb}$ and $\mathrm{Cs}$, the inter $p$-orbital hybridization with chalcogen anions is prevailing, shifting 
VBMs from $\Gamma$ to the $M$ point. As a result, except for $\mathrm{Na}_{2} \mathrm{X}(\mathrm{X}=\mathrm{O}, \mathrm{S}$, Se and $\mathrm{Te}$ ), all DMMC MLs are indirect semiconductors. The inter $p$-orbital hybridization induced direct to indirect bandgap transition from $\mathrm{Na}_{2} \mathrm{X}$ to other $\mathrm{A}_{2} \mathrm{X}$ MLs has also been confirmed by the PBE method, as shown in SI Table S2 and SI Figure S7.

As summarized in Fig. 3r., the energy gaps of DMMC MLs range from $1 \mathrm{eV}$ to $3 \mathrm{eV}$, covering the near-infrared $\left(\mathrm{K}_{2} \mathrm{O}, \mathrm{Rb}_{2} \mathrm{O}\right.$ and $\left.\mathrm{Cs}_{2} \mathrm{O} \mathrm{MLs}\right)$ and visible light regions, which are highly desirable for optoelectronic device applications. To evaluate the optical performance, the absorption coefficients of $\mathrm{Cs}_{2} \mathrm{O}$ and $\mathrm{Na}_{2} \mathrm{O}$ MLs are directly compared with bulk silicon [29]. As shown in Fig. 3d, $\mathrm{Na}_{2} \mathrm{O}$ ML shows absorption attenuation above $\sim 625 \mathrm{~nm}$, in consistent with the direct bandgap of $1.99 \mathrm{eV}$. Strikingly, indirect-bandgap $\mathrm{Cs}_{2} \mathrm{O}$ ML exhibits unrivaled cyan-to-red light absorption efficiency, which is nearly constant over the whole visible light wavelengths. Such unusual findings not only predict $\mathrm{Cs}_{2} \mathrm{O}$ MLs to be extraordinary optoelectronic materials, but also are rooted in the enormous, non-differentiable DOS in the vicinity of VBM, i.e. van Hove singularity. As shown in Fig. 3a and 3b, the top VBs of DMMC MLs, dominated by the localized $p$ orbitals of chalcogen anions, are distinctive by very flat dispersion in the momentum space. The inter $p$-orbital hybridization in $\mathrm{Cs}_{2} \mathrm{O} \mathrm{ML}$ drastically reduces the VB energy dispersion, which effectively enhances DOS near the VBM and creates multiple saddle points in the vicinity of $\Gamma, K$ and $M$. This leads to a prominent extended van Hove singularity in the density of states [30]. It is also noteworthy that the inter $p$-orbital hybridization also greatly enhances DOS near the CBM, which contributes significantly to the extraordinary light absorption characteristics of $\mathrm{Cs}_{2} \mathrm{O}$ ML.

Charge carrier mobility is another critical parameter for the device performance of $2 \mathrm{D}$ material-based devices. As summarized in Table I as well as in SI Table S3 and S4, the mobility of DMMC MLs are largely asymmetric between electrons and holes due to the drastic difference in the energy dispersion between the CBs and VBs, producing highly asymmetric effective mass. Noticeably, $\mathrm{K}_{2} \mathrm{O}$ ML has the highest electron mobility of $1.87 \times 10^{4}$ $\mathrm{cm}^{2} \mathrm{~V}^{-1} \mathrm{~S}^{-1}$ along the $y$ direction. Such electron mobility is comparable to the hole mobility of few-layer BP [17, 31] and order of magnitude larger than that of $\mathrm{MoS}_{2}$ atomic layers [13. Large electron mobility in DMMC MLs may hold a great promise for applications in high-performance electronics.

We have noticed that with the same trigonal structure $\left(R \overline{3} m\right.$, No.166), monolayer $\mathrm{Tl}_{2} \mathrm{O}$ is predicted to be a semiconductor with a direct bandgap and highly anisotropic charge 
carrier mobilities up to $4.3 \times 10^{3} \mathrm{~cm}^{2} \mathrm{~V}^{-1} \mathrm{~s}^{-1}$ [32]. This is not surprising since $\mathrm{Tl}$ is one of the pseudo-alkali metals, in close resemblance to $\mathrm{K}$ both in the oxidation state and ionic radius.

\section{Tunable Magnetism by Hole doping.}

The aforementioned van Hove singularities in the top VB of DMMC MLs are associated with inherent electronic instability, when hole doping pushes the Fermi level approaching the divergent point. Taking $\mathrm{Cs}_{2} \mathrm{O}$ as an example, we find that the system undergoes a spontaneous ferromagnetic phase transition with a moderate hole doping of $n \sim 1.0 \times 10^{13}$ $\mathrm{cm}^{-2}$. In Fig. 4 a, we plot the magnetic moment and the spin polarization energy per doped hole carrier as a function of $n$. The latter represents the energy difference between the non-spin-polarized phase and the ferromagnetic phase. Clearly, the top VB becomes fully spin polarized by the critical hole doping, as manifested by the constant magnetic moment of $1 \mu_{B}$ per carrier above $1.0 \times 10^{13} \mathrm{~cm}^{-2}$. The spin polarization energy, which becomes -3.11 meV per carrier for a minimal hoel doping, monotonically decreases as a function of $n$, approving that the doping-induced ferromagnetic ordering is energy favourable.

Such spontaneous ferromagnetic ordering can be well understood by the Stoner model, which has been adopted to explain the so-called " $d 0$ ferromagnetism" in hole doped nitrides and oxides [33]. In this picture, ferromagnetism can spontaneously appear when the Stoner criterion $U * g\left(E_{f}\right)>1$ is satisfied, where $U$ is the exchange interaction strength and $g\left(E_{f}\right)$ is the DOS at the Fermi energy of non-magnetic state. In such condition, the energy gain by exchange interactions exceeds the loss in kinetic energy, and hence the system would favor a ferromagnetic ground state. We have confirmed that the $U * g\left(E_{f}\right)$ of $\mathrm{Cs}_{2} \mathrm{O}$ is always larger than one above the critical doping level, indicating that the huge DOS associated with the van Hove singularity plays a key role in the occurrence of ferromagnetism.

In Fig. $4 \mathrm{~b}$ and $4 \mathrm{c}$, we plot the spin-polarized DOS of $\mathrm{Cs}_{2} \mathrm{O}$ with a doping level of $n=5.1 \times 10^{14} \mathrm{~cm}^{-2}$. Compared with the undoped case (Fig. 3 $\mathrm{b}$ ), the corresponding spin splitting process causes a significant energy shit of $0.1 \mathrm{eV}$ between the spin-up and spindown bands, leaving the Fermi level cutting through only one spin channel. Such half-metal state allows fully spin-polarized transport, which is crucial for spintronics applications. It is fascinating that this half-metallic ferromagnetism can be reversibly switched on and off in $\mathrm{a} \sim 1 \mathrm{~nm}$ thick atomic layer without extrinsic dopant and defects. 
We have found that except for $\mathrm{Na}_{2} \mathrm{Te}$, the ferromagnetic transition can be triggered in all DMMC MLs by hole doping, ranging from $\sim 1.0 \times 10^{13} \mathrm{~cm}^{-2}$ for $\mathrm{Cs}_{2} \mathrm{O}$ to $8.0 \times 10^{15}$ $\mathrm{cm}^{-2}$ for $\mathrm{Na}_{2} \mathrm{Se}$ (see SI Figure S8-S11). The special case of $\mathrm{Na}_{2} \mathrm{Te}$ can be understood by insufficient DOS near the VBM, which is dominated by the $5 p$ orbitals of Te. Due to much lower electron negativity, the $5 p$ orbitals of Te are more delocalized than the other chalcogen elements. Consequently, the high dispersion of Te-5p reduce DOS near the VBM of $\mathrm{Na}_{2} \mathrm{Te}$ (SI Fig. S8), invalidating the Stoner criterion. We further evaluated the doping dependent Curie temperature $\left(T_{c}\right)$ of DMMC MLs using the Heisenberg model. As shown in Fig. $4 \mathrm{~d}$, using $n=5.0 \times 10^{14} \mathrm{~cm}^{-2}$ as a reference, which can be readily induced by ionic liquid gating or lithium glass gating [16, 34, $\mathrm{RT}$ ferromagnetism is achievable in $\mathrm{K}_{2} \mathrm{O}$ and $\mathrm{Na}_{2} \mathrm{O}$ MLs (SI Figure S12). For $\mathrm{Cs}_{2} \mathrm{O}$, the corresponding $T_{c}$ is $\sim 200 \mathrm{~K}$, which is nearly five times higher than the recently reported ferromagnetic $\mathrm{CrI}_{3}$ and $\mathrm{Cr}_{2} \mathrm{Ge}_{2} \mathrm{Te}_{6}$ [20, 21].

\section{CONCLUSIONS}

In summary, we have reported a new family of 1T-TMDC structured dialkali-metal monochalcogenides (DMMCs), which show fascinating physical properties and great device application promises in the 2D limit. Distinctively, DMMC MLs are characterized by large electron mobility, e.g. $1.87 \times 10^{4} \mathrm{~cm}^{2} \mathrm{~V}^{-1} \mathrm{~S}^{-1}$ for $\mathrm{K}_{2} \mathrm{O}$ and $5.42 \times 10^{3} \mathrm{~cm}^{2} \mathrm{~V}^{-1} \mathrm{~S}^{-1}$ for $\mathrm{Na}_{2} \mathrm{O}$, and by very flat VBs with rather weak energy dispersion. The latter are responsible for the formation of extended van Hove singularities with extremely high DOS just below the VB edge of DMMC MLs. By introducing moderate hole doping, these DMMC MLs are subjected to strong electronic instabilities induced by electron-electron interaction, which ultimately leads to spontaneous ferromagnetic phase transitions where the top VBs becomes fully spin-polarized. Using heavy doping methods of ion liquid and solid gating, we can readily increase the $T_{c}$ and switch on and off the magnetism of DMMC MLs even at room temperature.

Although alkali compounds are not very stable in air by reacting with ambient moisture, the excellent dynamical and thermal stability allows DMMC MLs to be exfoliated and encapsulated in glove box environment, which is now routine research facilities for studying air-sensitive 2D materials [16, 17, 19 21]. It is also feasible to grow DMMC MLs by molecular beam epitaxy or by chemical vapor deposition, with abundant options of single crystal 
substrates of $1 \mathrm{~T}$ - or 2H-TMDCs. By forming heterostructure with high electron affinity main-group MDCs, such as $\mathrm{SnSe}_{2}$, DMMC MLs may become effectively hole doped by interfacial charge transferring [24], which would provide the opportunity for in-situ studying the gate-tunable ferromagnetism in DMMC MLs by scanning probe microscope.

[1] K. S. Novoselov, A. K. Geim, S. V. Morozov, D. Jiang, Y. Zhang, S. V. Dubonos, I. V. Grigorieva, and A. A. Firsov, "Electric field effect in atomically thin carbon films," Science 306, 666-669 (2004)

[2] K. S. Novoselov, A. K. Geim, S. V. Morozov, D. Jiang, M. I. Katsnelson, I. V. Grigorieva, S. V. Dubonos, and A. A. Firsov, "Two-dimensional gas of massless Dirac fermions in graphene," Nature 438, 197-200 (2005).

[3] Y. B. Zhang, Y. W. Tan, H. L. Stormer, and P. Kim, "Experimental observation of the quantum Hall effect and Berry's phase in graphene," Nature 438, 201-204 (2005).

[4] F. Zhu, W. Chen, Y. Xu, C. Gao, D. Guan, , C. Liu, D. Qian, S. C. Zhang, and J. F. Jia, "Epitaxial growth of two-dimensional stanene," Nature Mater. 14, 1020-1025 (2015).

[5] P. Vogt, P. De Padova, C. Quaresima, J. Avila, E. Frantzeskakis, , M. C. Asensio, A. Resta, B. Ealet, and G. Le Lay, "Silicene: Compelling experimental evidence for graphenelike twodimensional silicon," Phys. Rev. Lett. 108, 155501 (2012).

[6] C. C. Liu, W. X. Feng, and Y. G. Yao, "Quantum spin Hall effect in silicene and twodimensional germanium," Phys. Rev. Lett. 107, 076802 (2011).

[7] Y. H. Lu, D. Zhou, G. Q. Chang, S. Guan, W. G. Chen, Y. Z. Jiang, J. Z. Jiang, X. S. Wang, SY. A Yang, Y. P. Feng, Y. dKawazoe, and H. Lin, "Multiple unpinned Dirac points in group-Va single-layers with phosphorene structure," Npj. Comput. Mater. 2, 16011 (2016).

[8] K. Lee, S. W. Kim, Y. Toda, S. Matsuishi, and H. Hosono, "Dicalcium nitride as a twodimensional electride with an anionic electron layer," Nature 494, 336-340 (2013).

[9] M. Khazaei, M. Arai, T. Sasaki, C. Chung, N. S. Venkataramanan, M. Estili, Y. Sakka, and Y. Kawazoe, "Novel electronic and magnetic properties of twodimensional transition metal carbides and nitrides," Adv. Funct. Mater. 23, 2185-2192 (2013).

[10] G. R. Bhimanapati, Z. Lin, V. Meunier, Y. Jung, J. Cha, S. Das, D. Xiao, Y. Son, M. S. Strano, V. R. Cooper, L. Liang, S. G. Louie, W. Ringe, E. andZhou, S. S. Kim, R. R. Naik, B. G. 
Sumpter, H. Terrones, F. Xia, Y. Wang, J. Zhu, D. Akinwande, N. Alem, J. A. Schuller, R. E. Schaak, M. Terrones, and J. A. Robinson, "Recent advances in two-dimensional materials beyond graphene," ACS Nano 9, 11509-11539 (2015).

[11] A. K. Geim and I. V. Grigorieva, "Van der Waals heterostructures," Nature 499, 419-425 (2013).

[12] K. F. Mak, C. Lee, J. Hone, J. Shan, and T. F. Heinz, "Atomically thin $\mathrm{MoS}_{2}$ : A new direct-gap semiconductor," Phys. Rev. Lett. 105, 136805 (2010).

[13] B. Radisavljevic, A. Radenovic, J. Brivio, V. Giacometti, and A. Kis, "Single-layer $\mathrm{MoS}_{2}$ transistors," Nature Nanotech. 6, 147-150 (2011).

[14] Y. Zheng, J. Chen, M. F. Ng, H. Xu, Y. P. Liu, A. Li, S. J. O’Shea, T. Dumitrică, and K. P. Loh, "Quantum mechanical rippling of a $\mathrm{MoS}_{2}$ monolayer controlled by interlayer bilayer coupling," Phys. Rev. Lett. 114, 065501 (2015).

[15] G. Wang, A. Chernikov, M. M. Glazov, T. F. Heinz, X. Marie, T. Amand, and B. Urbaszek, "Colloquium: Excitons in atomically thin transition metal dichalcogenides," Rev. Mod. Phys. 90, $021001(2018)$.

[16] L. Li, Y. Yu, G. J. Ye, Q. Ge, X. Ou, H. Wu, D. Feng, X. H. Chen, and Y. B. Zhang, "Black phosphorus field-effect transistors," Nature Nanotech. 9, 372-377 (2014).

[17] L. K. Li, F. Y. Yang, G. J. Ye, Z. C. Zhang, Z. W. Zhu, W. K. Lou, X. Y. Zhou, L. Li, K. Watanabe, T. Taniguchi, K. Chang, Y. Y. Wang, X. H. Chen, and Y. B. Zhang, "Quantum Hall effect in black phosphorus two-dimensional electron system," Nature Nanotech. 11, 592$596(2016)$.

[18] T. Cao, Z. Li, and S. G. Louie, "Tunable magnetism and half-metallicity in hole-doped monolayer GaSe," Phys. Rev. Lett. 114, 236602 (2015).

[19] D. A. Banhurin, A. V. Tyurnina, G. L. Yu, A. Mishchenko, V. Zolyomi, Sergey V. Morozov, R. K. Kumar, R. V. Gorbachev, Z. R. Kudrynskyi, S. Pezzini, Z. D. Kovalyuk, U. Zeitler, K. S. Novoselov, A. Patan, L. Eaves, I. V. Grigorieva, V. I. Fal'ko, A. K. Geim, and Y. Cao, "High electron mobility, quantum Hall effect and anomalous optical response in atomically thin InSe," Nature Nanotech. 12, 223-227 (2017).

[20] C. Gong, L. Li, Z. Li, H. Ji, A. Stern, Y. Xia, T. Cao, W. Bao, C. Wang, Y. Wang, Z. Q. Qiu, R. J. Cava, S. G. Louie, J. Xia, and X. Zhang, "Discovery of intrinsic ferromagnetism in two-dimensional van der Waals crystals," Nature 546, 265-269 (2017). 
[21] B. Huang, G. Clark, E. Navarro-Moratalla, D. R. Klein, R. Cheng, K. L. Seyler, D. Zhong, E. Schmidgall, M. A. McGuire, D. H. Cobden, W. Yao, D. Xiao, P. Jarillo-Herrero, and $\mathrm{X} . \mathrm{Xu}$, "Layer-dependent ferromagnetism in a van der Waals crystal down to the monolayer limit," Nature 546, 270-273 (2017).

[22] L. C. Gomes and A. Carvalho, "Phosphorene analogues: Isoelectronic two-dimensional groupIV monochalcogenides with orthorhombic structure," Phys. Rev. B 92, 085406 (2015).

[23] M. Wu and X. C. Zeng, "Intrinsic ferroelasticity and/or multiferroicity in two-dimensional phosphorene and phosphorene analogues," Nano Lett. 16, 3236-3241 (2016).

[24] Z. Wang, C. Fan, Z. X. Shen, C. Hua, Q. Hu, F. Sheng, Y. H. Lu, H. Fang, Z. Qiu, J. Lu, Z. Liu, W. Liu, Y. Huang, Z. A. Xu, D. W. Shen, and Y. Zheng, "Defects controlled hole doping and multivalley transport in SnSe single crystals," Nature Commun. 9, 47 (2018).

[25] M. Naguib, O. Mashtalir, J. Carle, V. Presser, J. Lu, L. Hultman, Y. Gogotsi, and M. W. Barsoum, "Two-dimensional transition metal carbides," ACS Nano 6, 1322-1331 (2012),

[26] K. Tsai, P. M. Harris, and E. N. Lassettre, "The crystal structure of cesium monoxide," J. Phys. Chem. 60, 338-344 (1956).

[27] J. A. Wilson, F. J. Di Salvo, and S. Mahajan, "Charge-density waves and superlattices in the metallic layered transition metal dichalcogenides," Adv. Phys. 50, 1171-1248 (2001).

[28] R. Zacharia, H. Ulbricht, and T. Hertel, "Interlayer cohesive energy of graphite from thermal desorption of polyaromatic hydrocarbons," Phys. Rev. B 69, 155406 (2004).

[29] M. A. Green and M. J. Keevers, "Optical properties of intrinsic silicon at 300 K," Prog. Photovolt: Res. Appl. 3, 189-192 (1995).

[30] K. Gofron, J. C. Campuzano, A. A. Abrikosov, M. Lindroos, A. Bansil, H. Ding, D. Koelling, and B. Dabrowski, "Observation of an "extended" van Hove singularity in $\mathrm{YBa}_{2} \mathrm{Cu}_{4} \mathrm{O}_{8}$ by ultrahigh energy resolution angle-resolved photoemission," Phys. Rev. Lett. 73, 3302 (1994).

[31] J. Qiao, X. Kong, Z. Hu, F. Yang, and W. Ji, "High-mobility transport anisotropy and linear dichroism in few-layer black phosphorus," Nature Commun. 5, 4475 (2014).

[32] Y. D. Ma, A. Kuc, and T. Heine, "Single-layer $\mathrm{Tl}_{2} \mathrm{O}$ : A metal-shrouded 2D semiconductor with high electronic mobility," J. Am. Chem. Soc. 139, 11694-11697 (2017).

[33] H. Peng, H. J. Xiang, S. H. Wei, S. S. Li, J. B. Xia, and J. B. Li, "Origin and enhancement of hole-induced ferromagnetism in first-row $d^{0}$ semiconductors," Phys. Rev. Lett. 102, 017201 $(2009)$. 
[34] B. Lei, J. H. Cui, Z. J. Xiang, C. Shang, N. Z. Wang, G. J. Ye, X. G. Luo, T. Wu, Z. Sun, and X. H. Chen, "Evolution of high-temperature superconductivity from a low- $T_{c}$ phase tuned by carrier concentration in FeSe thin flakes," Phys. Rev. Lett. 116, 077002 (2016).

\section{ACKNOWLEDGMENTS}

This work is supported by the National Key R\&D Program of the MOST of China (Grant Nos. 2016YFA0300204 and 2017YFA0303002), and the National Science Foundation of China (Grant Nos. 11574264 and 61574123). Y.Z. acknowledges the funding support from the Fundamental Research Funds for the Central Universities and the Thousand Talents Plan.

\section{COMPETING FINANCIAL INTERESTS:}

The authors declare no competing financial interests. 
FIGURES 


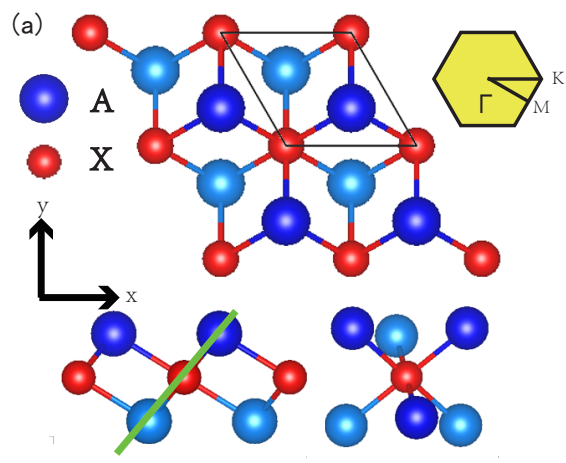

(b)

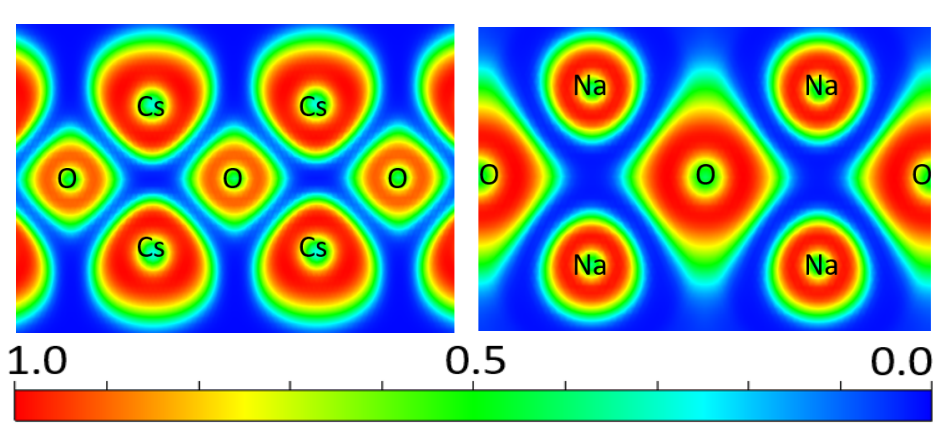

FIG. 1. (a) Structure of DMMC MLs. The upper inset is the first Brillouin zone. Each chalcogen anion is surrounded by six alkali cations, forming $\mathrm{XA}_{6}$ octahedron. (b-c) Normalized electron localization function (ELF) of $\mathrm{Cs}_{2} \mathrm{O}$ and $\mathrm{Na}_{2} \mathrm{O}$, respectively. ELF $=1$ (red) and 0 (blue) indicate accumulated and vanishing electron densities, respectively. The 2D ELF plane is defined by the green line in Fig. 1a. 
(a)

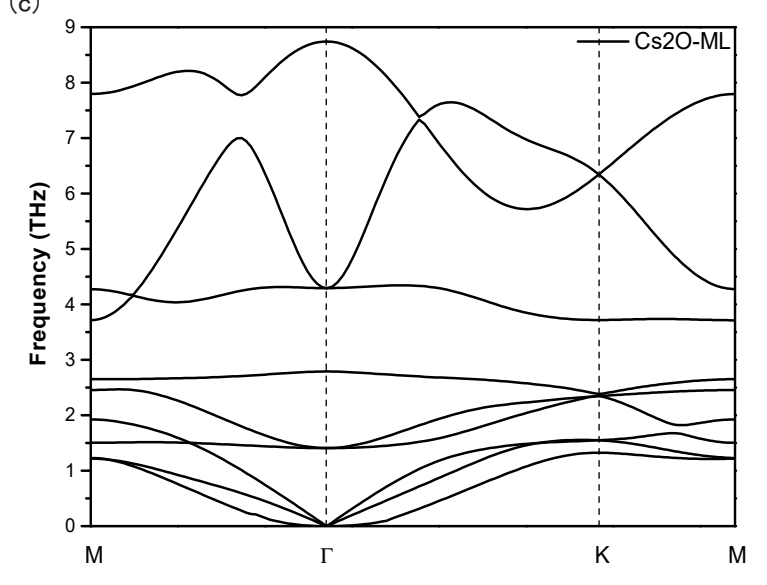

(b)
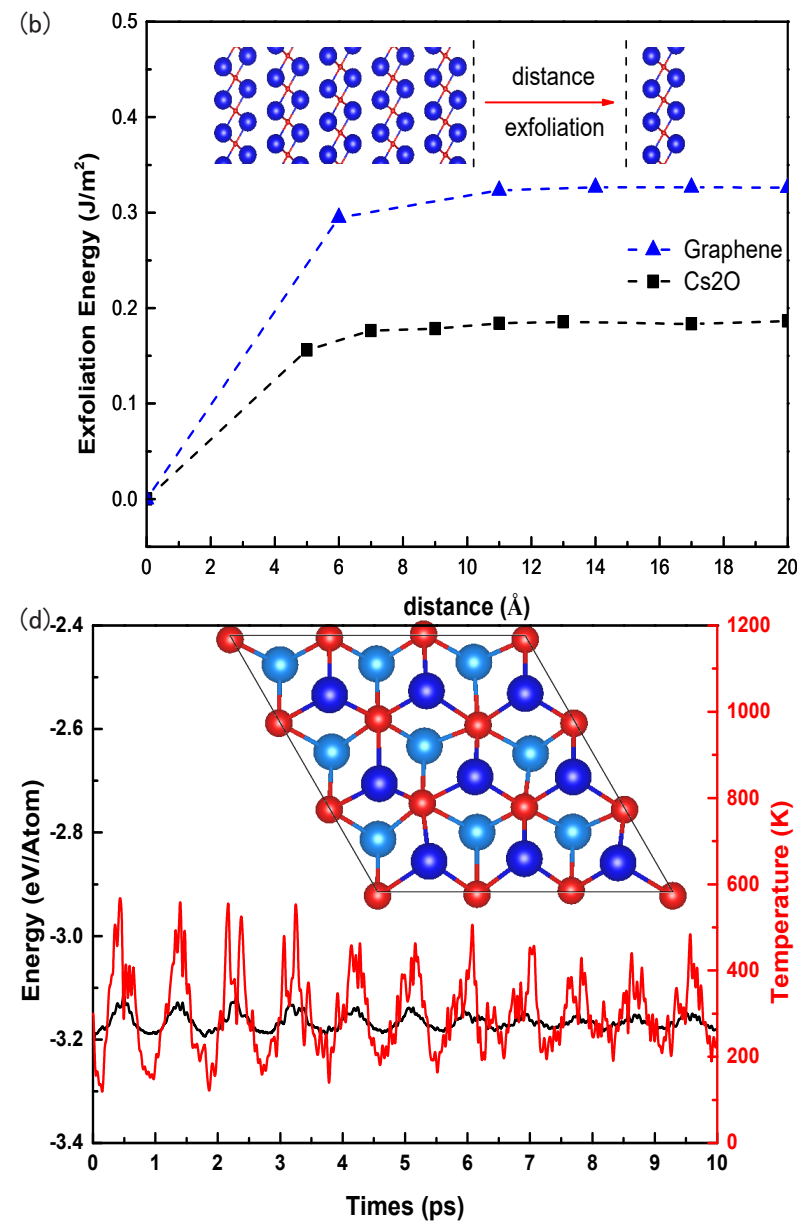

FIG. 2. Stability and Micromechanical Exfoliation. (a) Interlayer differential charge density of $\mathrm{Cs}_{2} \mathrm{O}$ bulk, graphite and $\mathrm{MoS}_{2}$ bulk. (b) Calculated exfoliation energy of $\mathrm{Cs}_{2} \mathrm{O}$ ML, in comparison with graphene (blue triangles). (c) The phonon spectrum of $\mathrm{Cs}_{2} \mathrm{O}$ ML. (d) First-principle molecular dynamics simulations of $\mathrm{Cs}_{2} \mathrm{O}$ ML. The thermal stability of the supercell is evident by the convergence in the free energy. 


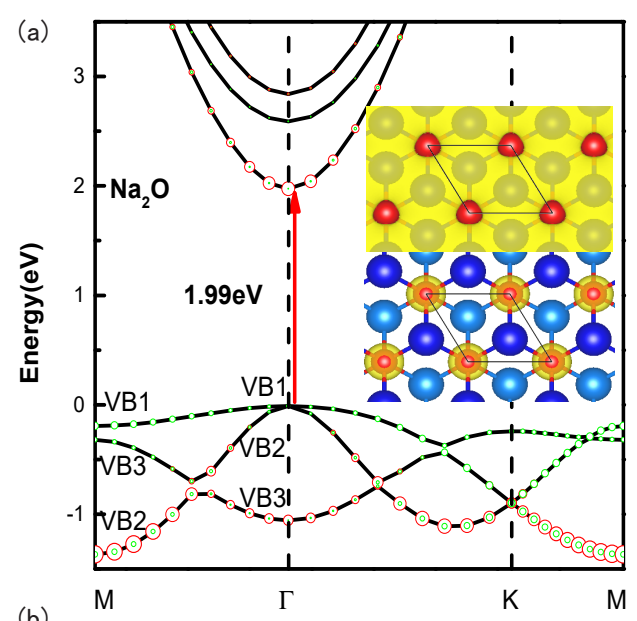

(b)

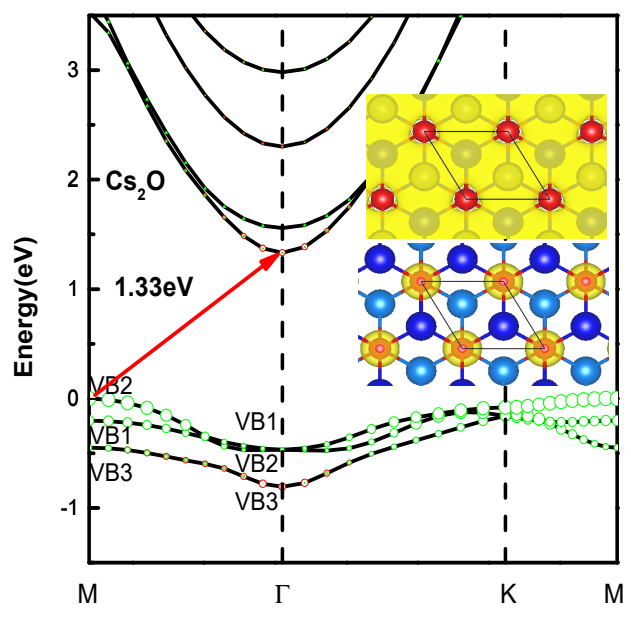

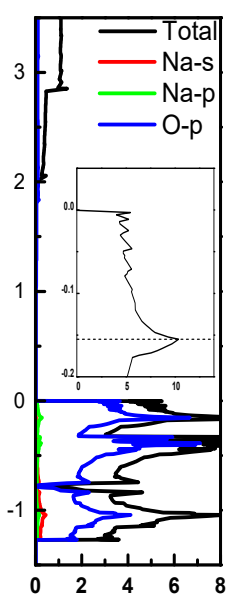

DOS(a.u.)

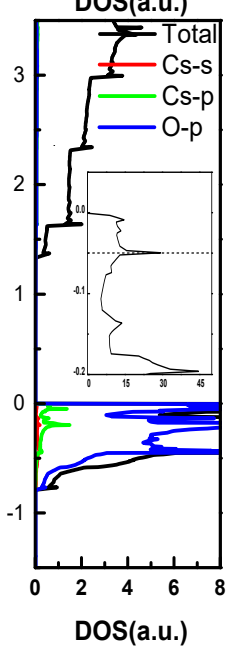

(c)

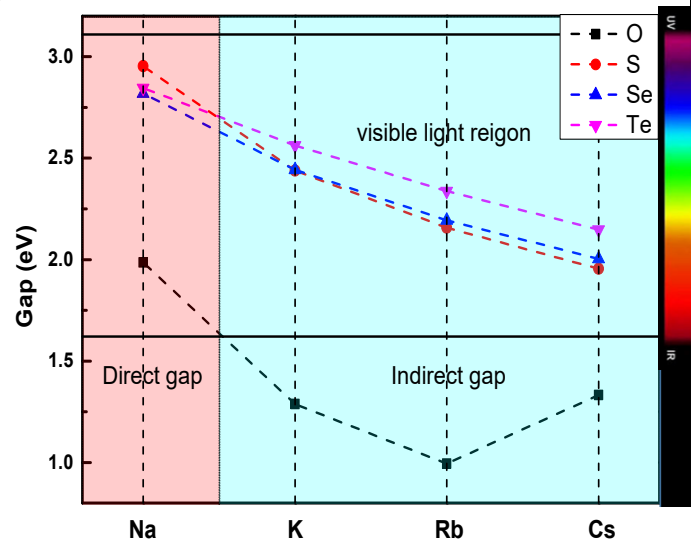

(d)

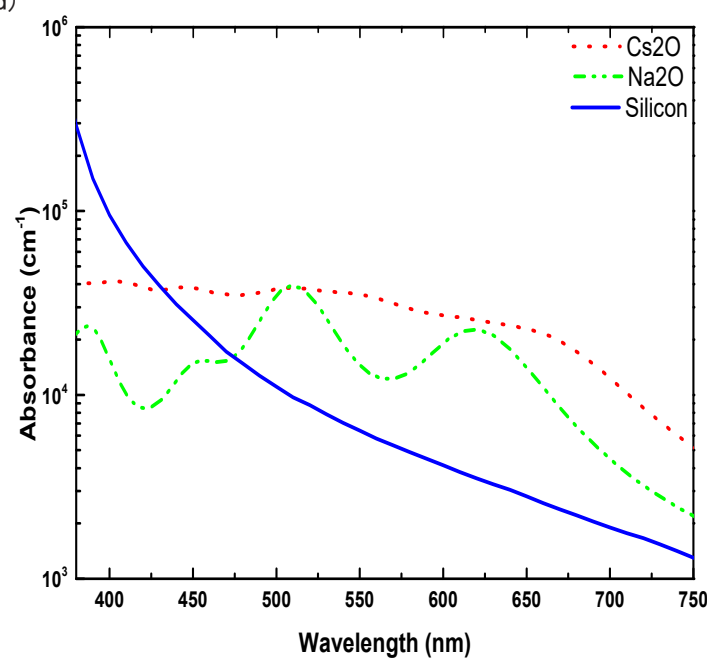

FIG. 3. Electronic Properties. Band structures and the corresponding DOS of $\mathrm{Na}_{2} \mathrm{O}$ ML (a) and $\mathrm{Cs}_{2} \mathrm{O}$ ML (b). The contributions of alkali $s$-orbital and $p$-orbital are indicated by red and green circles, respectively. The insets are the spatial distribution of the wave-functions for the VBM and CBM. (c) Energy gaps of all DMMC MLs. Strong inter p-orbital hybridization leads to the transition from direct bandgaps in $\mathrm{Na}_{2} \mathrm{X}$ to indirect gaps in other DMMC MLs. The gap values of DMMC MLs are mainly determined by the electron affinity of the anions. The results have been confirmed by both the HSE06 and PBE methods. (d) Extraordinary light absorbance of $\mathrm{Cs}_{2} \mathrm{O}$ and $\mathrm{Na}_{2} \mathrm{O}$ MLs, in comparison with silicon. 


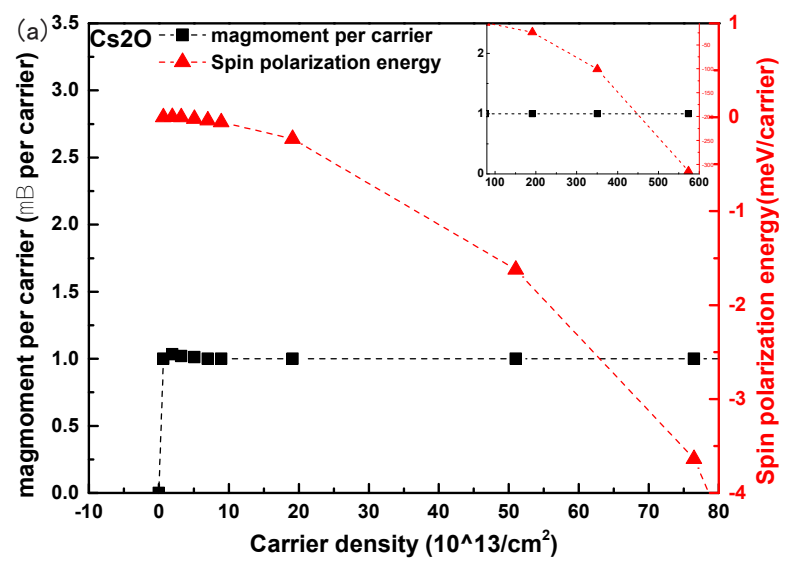

(c)

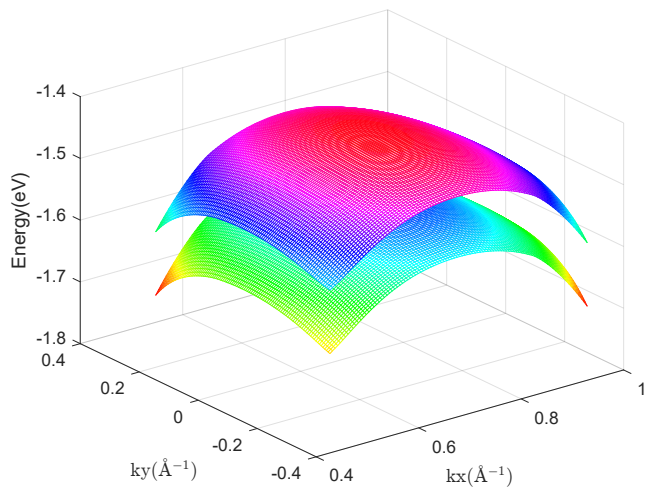

(b)

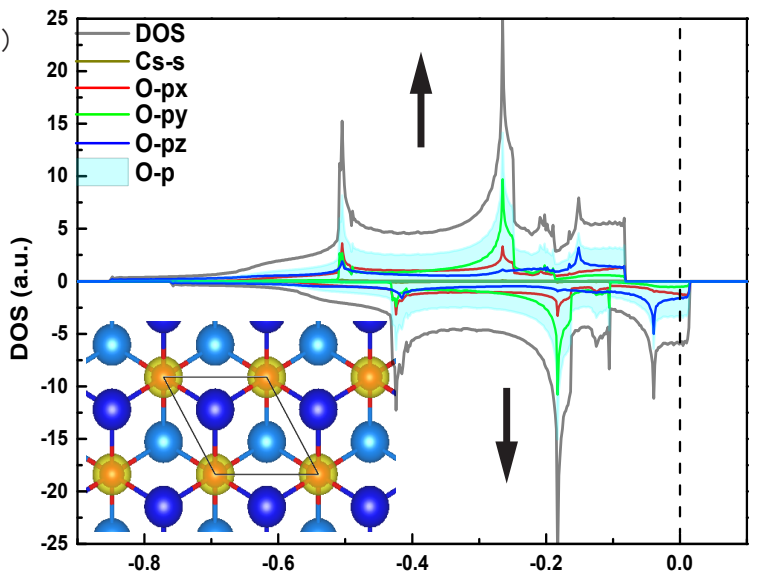

(d)

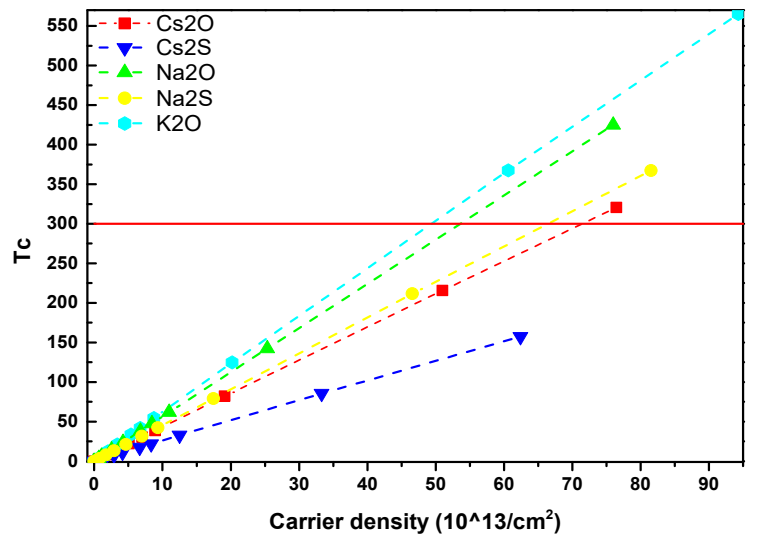

FIG. 4. Tunable Magnetism by Hole Doping. (a) Magnetic moment and spin polarization energy per carrier of $\mathrm{Cs}_{2} \mathrm{O}$ ML. Remarkably, spontaneous ferromagnetic phase transition is trigger by a moderate hole doping of $1.28 \times 10^{13} \mathrm{~cm}^{-2}$. (b) Spin-resolved DOS of $\mathrm{Cs}_{2} \mathrm{O}$ ML at $n=5 \times 10^{14}$ $\mathrm{cm}^{-2}$. The inset shows the spin density distribution, apparently locating at $\mathrm{O}$ atoms. (c) Spinsplitted top valence bands in the vicinity of $M$ points in $\mathrm{Cs}_{2} \mathrm{O} \mathrm{ML}\left(n=5 \times 10^{14} \mathrm{~cm}^{-2}\right)$. (d) Curie temperature verus hole doping density, showing the feasibility of RT ferromagnetism by heavy hole doping. Note that antiferromagnetic ordering in DMMC MLs is energetically higher in free energy than the ferromagnetic state. 
TABLES 
TABLE I. Mobility and effective mass along the $x$ and $y$ transport directions in four $\mathbf{C s}_{2} \mathbf{X}$ MLs and $\mathbf{K}_{2} \mathbf{O}$. We calculated the carrier mobility using a phonon-limited scattering model, which includes deformation potential $\left(\mathrm{E}_{1}\right)$ and elastic modulus $\left(\mathrm{C}_{2 D}\right)$ in the propagation direction of the longitudinal acoustic wave. Noticeably, $\mathrm{K}_{2} \mathrm{O}$ has the highest electron mobility.

\begin{tabular}{ccccc} 
Effective mass $\left(\mathrm{m}_{0}\right)$ & $\mathrm{m}_{h x}$ & $\mathrm{~m}_{h y}$ & $\mathrm{~m}_{e x}$ & $\mathrm{~m}_{e y}$ \\
\hline $\mathrm{Cs}_{2} \mathrm{O}$ & 5.36 & 4.71 & 0.63 & 0.84 \\
$\mathrm{Cs}_{2} \mathrm{~S}$ & 1.02 & 2.13 & 0.67 & 0.89 \\
$\mathrm{Cs}_{2} \mathrm{Se}$ & 0.95 & 1.99 & 0.61 & 0.81 \\
$\mathrm{Cs}_{2} \mathrm{Te}$ & 1.03 & 1.88 & 0.58 & 0.77 \\
$\mathrm{~K}_{2} \mathrm{O}$ & 1.84 & 11.2 & 0.90 & 0.67 \\
\hline Mobility $\left(\mathrm{cm}^{2} \mathrm{~V}^{-1} \mathrm{~S}^{-1}\right)$ & $\mu_{h x}$ & $\mu_{h y}$ & $\mu_{e x}$ & $\mu_{e y}$ \\
\hline $\mathrm{Cs}_{2} \mathrm{O}$ & $0.13 \times 10^{1}$ & $0.34 \times 10^{1}$ & $9.63 \times 10^{1}$ & $1.87 \times 10^{1}$ \\
$\mathrm{Cs}_{2} \mathrm{~S}$ & $0.29 \times 10^{1}$ & $0.36 \times 10^{1}$ & $6.99 \times 10^{1}$ & $1.10 \times 10^{2}$ \\
$\mathrm{Cs}_{2} \mathrm{Se}$ & $0.15 \times 10^{1}$ & $9.51 \times 10^{-1}$ & $5.60 \times 10^{1}$ & $4.33 \times 10^{1}$ \\
$\mathrm{Cs}_{2} \mathrm{Te}$ & $9.07 \times 10^{-1}$ & $6.95 \times 10^{-1}$ & $2.61 \times 10^{1}$ & $2.37 \times 10^{1}$ \\
$\mathrm{~K}_{2} \mathrm{O}$ & $0.46 \times 10^{1}$ & $2.53 \times 10^{-1}$ & $\mathbf{8 . 8 0} \times \mathbf{1 0}$ & $\mathbf{1 . 8 7} \times \mathbf{1 0}$ \\
\hline
\end{tabular}

